\title{
ELECTRONIC TRANSPORT PROPERTIES CHARACTERIZATION OF SILICON WAFERS BY MODULATED PHOTOREFLECTANCE
}

\author{
B.C. FORGET and D. FOURNIER \\ Laboratoire d'Instrumentation, Univ. Pierre \& Marie Curie, Paris VIESPCI, 10 nue Vauquelin, \\ F-75005 Paris, France
}

\begin{abstract}
We have used the photoreflectance technique to characterize electronical transport properties of silicon wafers. By using the intermediate frequency range $(1 \mathrm{kHz}$ to $100 \mathrm{kHz})$ and selecting a proper excitation wavelength, it is possible to facilitate the characterization. In particular we show that this technique permits the determination of surface recombination velocity.
\end{abstract}

\section{Introduction}

Photothermal techniques have largely contributed to thermal characterization and transport properties determination in semiconductors $/ 1-3 /$. In this paper, we use the intermediate frequency range (from a $1 \mathrm{kHz}$ to $100 \mathrm{kHz}$ ) to study the electronic transport properties of silicon wafers. This frequency range allows the observation of the passage from a thermal wave signal, at low frequencies, to a plasma wave signal, or Drude effect, at high frequencies.

This passage is characteristic of the electronic transport properties of a semiconductor since the heat is transferred to the lattice by the recombination of the photogenerated electron-hole plasma after its diffusion in the sample. We show in particular that this technique allows a non ambiguous determination of the surface recombination velocity. We also show that a proper choice of wavelength for the excitation, or pump beam, can greatly facilitate this determination.

\section{Theory of the photothermal effect in semiconductors}

Let us consider what happens when an incident excitation (pump), in our case a laser beam , is absorbed by a semiconductor. If the energy of the incident photons, $h v$, is greater than the gap energy, $\mathrm{E}_{\mathrm{g}}$, the absorption will generate a free-carrier plasma with an energy equal to the photon energy, hv. This absorbed energy will be returned to lattice as heat in a two step process. The electrons and hole will relax to the minimum of the conduction and valence band respectively, transferring the excess energy, $h v-E_{g}$, to the lattice as heat. This relaxation is generally very fast $\left(\approx 10^{-12} \mathrm{sec}\right)$ compared to the other phenomena involved and is considered instantaneous. Then the electron-hole plasma will diffuse until the pairs recombine after a lifetime, $\tau$, characteristic of the probability of pair recombination in the bulk of 
the sample. This recombination will transfer to lattice the remaining part of photon energy, which is the band gap energy, $\mathrm{E}_{\mathrm{g}}$.

Therefore to obtain the expression of the photothermal signal we must firstly solve the plasma diffusion equation. The plasma density obtained will then be used as a source term in the heat diffusion equation. However, in a semiconductor, the complex index of refraction is affected not only by the temperature rise, $\Delta T$, but also by the density of the electron-hole plasma photogenerated if the energy of the incident photons is greater than that of the gap through the Drude effect, $\Delta N$. We can express the variation of the optical reflectivity, $\Delta R$, as follows:

$$
\Delta \mathrm{R}=\frac{\partial \mathrm{R}}{\partial \mathrm{T}} \Delta \mathrm{T}+\frac{\partial \mathrm{R}}{\partial \mathrm{N}} \Delta \mathrm{N}
$$

The electron-hole plasma density contributes directly to the signal in addition to being a distributed heat source in the bulk of the sample. The relative amplitude of these two contributions is partly determined by the modulation frequency. At very high frequencies (typically in $\mathrm{MHz}$ domain), the observation period is too short to allow the plasma to recombine and produce heat: the modulation of the reflectivity is mainly due to the Drude effect. In Silicon, the coefficients $\partial R / \partial N$ and $\partial R / \partial T$ are opposite in sign, making easy to observe the passage from one regime to the other $/ 4 /$, and thus characterize the electronic transport properties.

In our experimental setup, the pump beam radius is smaller than the thermal and electronic diffusion lengths, therefore the monodimensional solution of the diffusion equations is not sufficient to interpret our experimental data. To obtain the three dimensional expression we make use of integral transformations and the axial symmetry of the problem. By replacing the modulated source with a unit source and successively using the Hankel transformation and the Laplace transformation we can use the same simple procedure as for the 1-D calculation to obtain a solution in the Hankel domain $/ 5 \%$. This calculation leads to a somewhat complicated expression /6/. Finally we use a numerical calculation of the inverse Hankel transform $/ 7 /$ to obtain the expression of the photoreflectance in the real domain.

\section{Influence of excess energy}

From the theoretical discussion above we see that the photothermal signal can be expressed as a combination of three terms: the Drude effect, or plasma term, the thermal term due to the recombination of the electron-hole plasma and finally the thermal term due to the rapid thermalization of the energy in excess of the band gap. Contrarily to the first two terms, the effect of the excess energy thermalization is in no way characteristic of the electronic transport properties of the semiconductor. It therefore seems advantageous to minimize this excess energy term by using a laser with a photon energy close to the band gap.

It is important to note that difference of the signal as a function of pump modulation frequency for the three different wavelengths is due not only to the reduction of the excess energy but also to the variation of the optical absorption coefficient, $\alpha$, that is dependant of the incident wavelength. 


\section{Experimental Setup}

We have used a classical photoreflectance setup (shown schematically in figure \#1) with a $80 \mu \mathrm{m}$ diameter excitation induced by either a $450 \mathrm{~mW}$ Art laser $(\lambda=514 \mathrm{~nm})$ and a a 400 $\mathrm{mW}$ dye laser using Kitton Red dye $(\lambda=$ $625 \mathrm{~nm})$. This excitation beam is modulated by an acousto-optic modulator from $1 \mathrm{kHz}$ to $100 \mathrm{kHz}$. The induced variation of the reflected $780 \mathrm{~nm}$ diode laser is detected by a silicon photodiode, or avalanche photodiode, connected to a lock-in amplifier. The probe beam has a $35 \mu \mathrm{m}$ diameter and is focussed on the same spot as the pump beam. We have achieved a sensitivity of $10^{-6}$ in $\Delta R / R$ with this set-up.

\section{Results and Discussion}



Figure1: Experimental Setup

In order to test the validity of our experimental technique and theoretical model, we have used a reference sample of aluminium which produces a purely thermal signal, easier to analyze. Experimental data shown in figure \#2 is in good accordance with the theoretical model $/ 5 /$.

(a.u.)

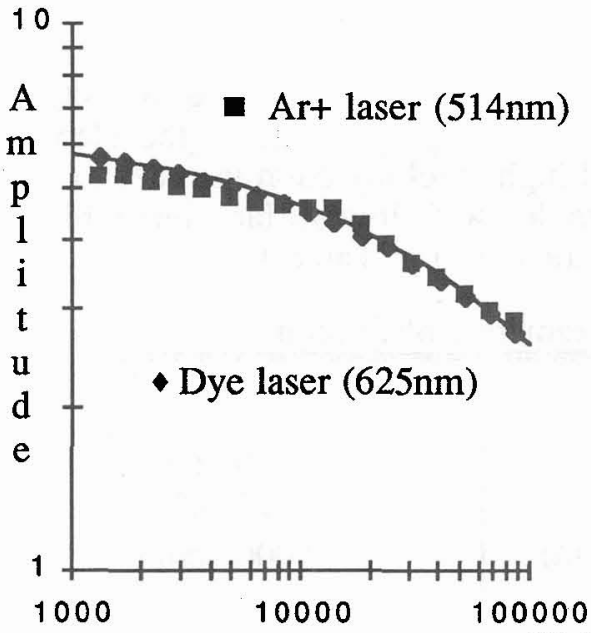

Modulation Frequency

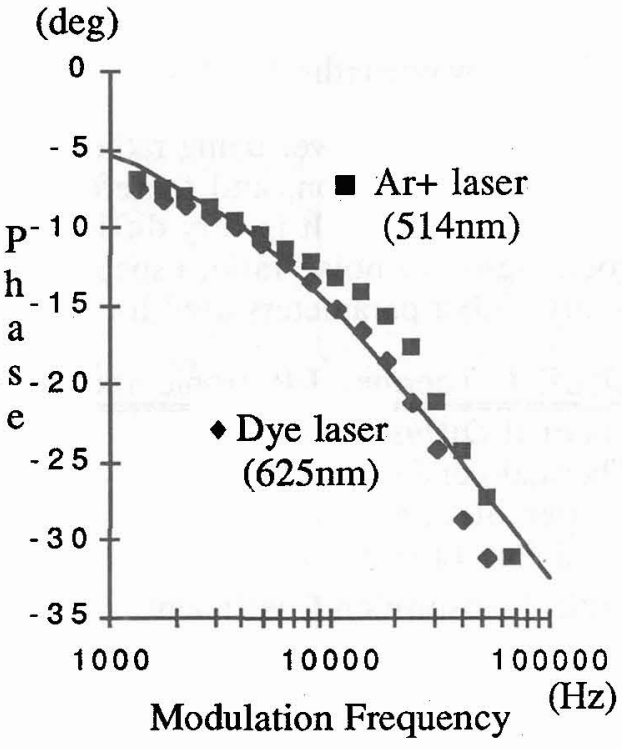
) and experimental data $(\bullet, \mathbf{m})$ of the amplitude and phase of the photoreflectance signal for the reference sample of Al. 
Figure \#3 shows the amplitude and phase of the photoreflectance signal for a 300 $\mu \mathrm{m}$ thick, n-type silicon wafer, with theoretical calculation, for the two excitation beams. The surface of this sample had been polished and, as expected, the surface recombination velocity is very low $(\mathrm{s}=200 \mathrm{~cm} / \mathrm{s})$. We can clearly see that using the $625 \mathrm{~nm}$ excitation permits us detect the plasma regime (the rise in amplitude and a steeper phase shift) at lower frequencies than with the $514 \mathrm{~nm}$ excitation. Again experimental data and theoretical calculations are in good agreement.
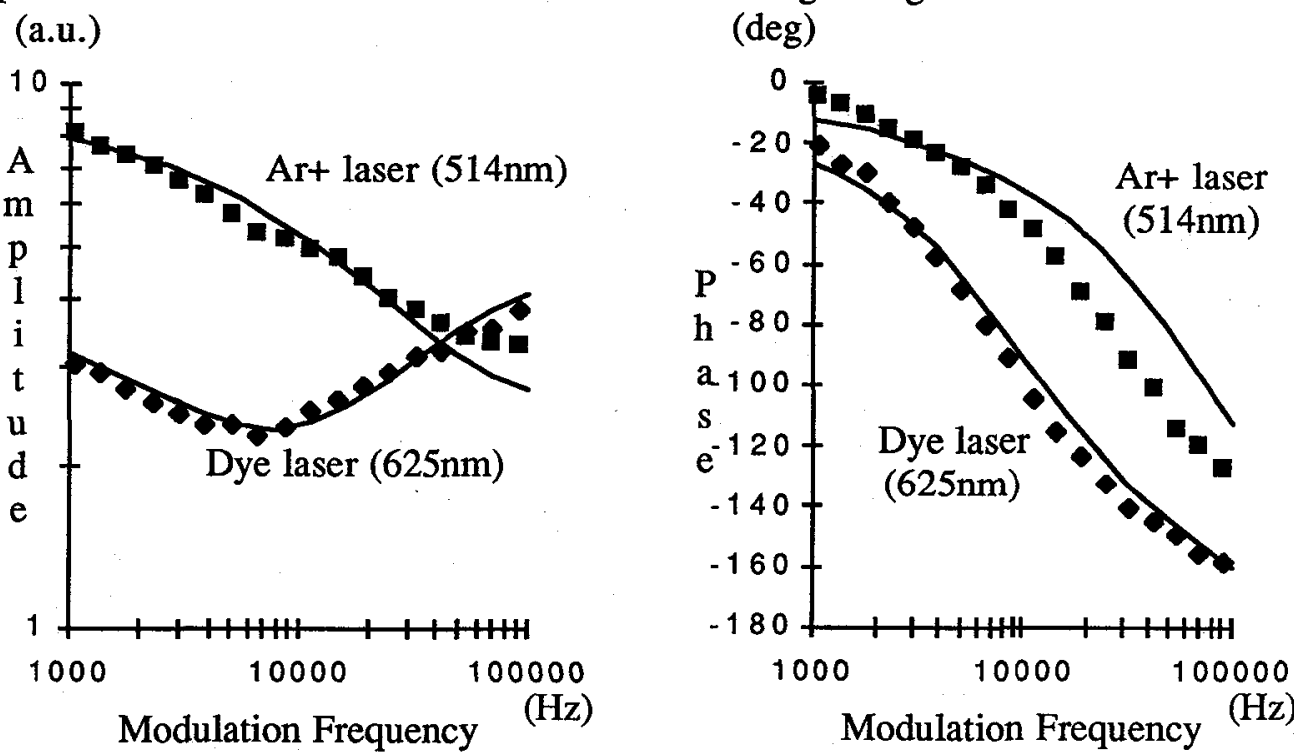

Figure 3: Theoretical calculation $(-)$ and experimental data $(\bullet, \infty)$ of the amplitude and phase of the photoreflectance signal for two excitation wavlengths.

The incident power being rather tightly focussed, we find ourselves in a situation of high level injection, and therefore we use the ambipolar value of the electronic diffusivity: $14 \mathrm{~cm}^{2} / \mathrm{s}$. It is very difficult to avoid high level injection in order to obtain a good signal to noise ratio, especially for samples with low surface recombination velocity. Other parameters used for calculation are shown in Table 1.

TABLE 1: Thermal, Electronic and Optical Parameters of Silicon

\begin{tabular}{|l|l|r|}
\hline \hline Thermal Diffusivity & Dth & $0.9 \mathrm{~cm}^{2} \mathrm{~s}^{-1}$ \\
Thermal conductivity & $\mathrm{k}$ & $1.5 \mathrm{~W} \mathrm{~K}^{-1} \mathrm{~cm}^{-1}$ \\
Carrier lifetime & $\tau$ & $50 \mu \mathrm{s}$ \\
Band Gap Energy & $\mathrm{E}_{\mathrm{g}}$ & $1.12 \mathrm{eV}$ \\
Optical Absorption Coefficient & $\alpha(514 \mathrm{~nm})$ & $15000 \mathrm{~cm}^{-1}$ \\
& $\alpha(625 \mathrm{~nm})$ & $3950 \mathrm{~cm}^{-1}$ \\
Incident photon Energy & $\mathrm{hv}(514 \mathrm{~nm})$ & $2.42 \mathrm{eV}$ \\
& $\mathrm{hv}(625 \mathrm{~nm})$ & $1.9 \mathrm{eV}$ \\
Plasma reflectance coefficient & $1 / \mathrm{Ro} \partial \mathrm{R} / \partial \mathrm{N}$ & $-1.66 \times 10-22 \mathrm{~cm}^{3}$ \\
Thermal reflectance coefficient & $1 / \mathrm{Ro} \partial \mathrm{R} / \partial \mathrm{T}$ & $1.5 \times 10-4 \mathrm{~K}^{-1}$ \\
\hline
\end{tabular}


We repeated the experiment on a sample whose surface had been scratched. As expected, the surface recombination velocity is greatly increased by this damage $(\mathrm{s}=4000 \mathrm{~cm} / \mathrm{s})$. In this case, the difference in signal (shown in Figure \#4) obtained with the two lasers is less apparent. In the case of high surface recombination velocity, the relative importance of the thermal contribution compared to that of the plasma contribution is increased and therefore dominant throughout the frequency range of our experiments.

(a.u.)

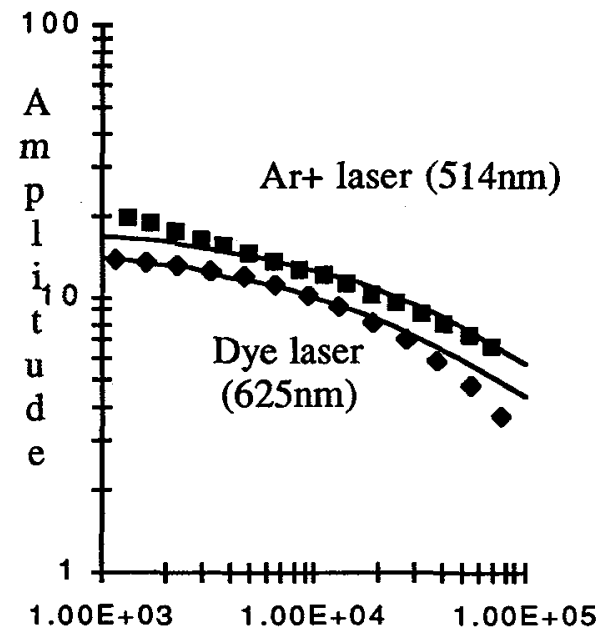

Modulation Frequency

$(\mathrm{Hz})$

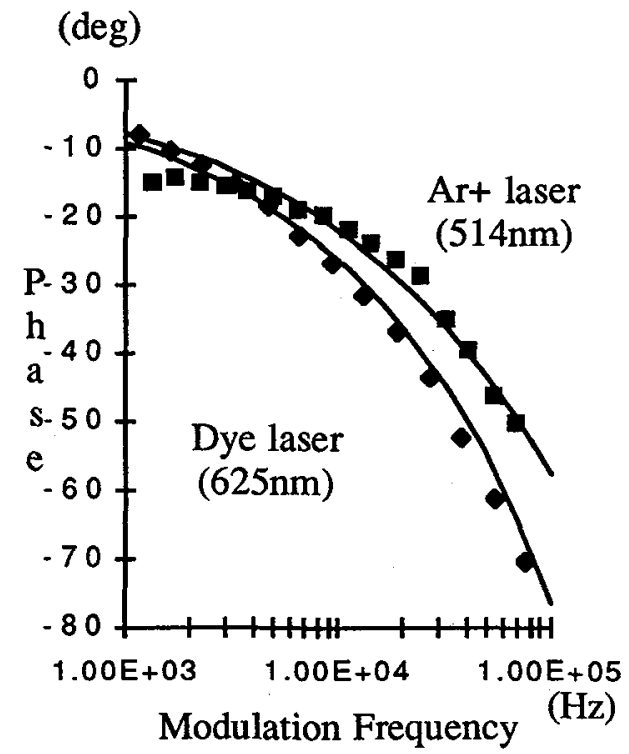

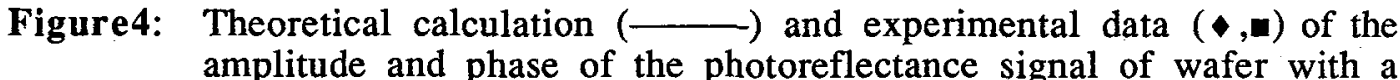
"scratched" surface for two excitation wavlengths.

We are now improving this technique by switching to a Styryl-9 dye which will give us an $830 \mathrm{~nm}$ excitation. At $625 \mathrm{~nm}$, incident photons have still an energy greater than the band gap of silicon (almost two times the band gap) so the excess energy thermal contribution is still very important. The infrared excitation will reduce even more this contribution, permitting easier determination of electronic transport properties at lower frequencies.

\section{References}

/1/ W.L.Smith, A.Rosencwaig and D.L.Willemborg, Appl.Phys.Lett. 47 (1985), 584.

/2/ A.Pinto Neto, H.Vargas, N.F.Leite and L.C.M.Miranda, Phys.Rev. (1990), 9971.

13/ F.A.MacDonald, D.Guidotti, and T.M.DelGiudice, from: "Review of Progress in Quantitative Nondestructive Evaluation" Vol. 6B ed. by D.O. Thompson and D.E. Chimenti , Plenum (1987), 1361.

14/ J.Pelzl, D.Fournier and A.C.Boccara, 5th International topical Meeting on Photoacoustic and Photothermic Phenomena (Heildelberg 1987).

15/ D.Fournier and A.C.Boccara, Photothermal Investigation of Solids: Basic Physical Principles, Academic Press (1988), 35. 
16/ N.Mikoshiba, H.Nakamura and K.Tsubouchi, Ultrasonics Symposium Proceedings, IEEE New York (1982), 580.

The model presented here omits the rapid thermalization of energy in excess of the band edge. See $13 /$.

/7I S.M.Candel, Computer Phys. Comm. 23 (1981), 343. 